BMJ Open

Diabetes

Research

\& Care

\title{
Alterations in plantar vessel blood flow in patients with mild diabetic peripheral neuropathy
}

Qiang Zhou, ${ }^{1,2}$ Zhihui Qian, ${ }^{1}$ Maoguang Yang, ${ }^{3}$ Jing Liu, ${ }^{1}$ Jianan Wu, ${ }^{1}$ Luquan Ren, ${ }^{1}$ Lei Ren (iD) ${ }^{1,4}$

\section{ABSTRACT}

Yang M, et al. Alterations in plantar vessel blood flow in patients with mild diabetic peripheral neuropathy. BMJ Open Diab Res

Care 2022;10:e002492.

doi:10.1136/

bmjdrc-2021-002492

\section{- Additional supplemental} material is published online only. To view, please visit the journal online (http://dx.doi. org/10.1136/bmjdrc-2021002492).

Received 15 July 2021 Accepted 10 December 2021

Check for updates

\section{(c) Author(s) (or their} employer(s)) 2022. Re-use permitted under CC BY-NC. No commercial re-use. See rights and permissions. Published by BMJ.

${ }^{1}$ Key Laboratory of Bionic Engineering, Ministry of Education, Jilin University, Changchun, China

${ }^{2}$ Cadre's Ward, The First Hospital of Jilin University, Changchun, China

${ }^{3}$ Department of Endocrinology, The Second Hospital of Jilin University, Changchun, China ${ }^{4}$ School of Mechanical, Aerospace and Civil Engineering, University of Manchester, Manchester, UK

Correspondence to Dr Lei Ren;

lei.ren@manchester.ac.uk and Dr Jing Liu; jingliu@jlu.edu.cn
Introduction Early identification and treatment of diabetic peripheral neuropathy (DPN) are crucial. Presently, the mechanism of DPN is not very clear, and there are inconclusive conclusions about the influencing factors of vascular dynamic characteristics in DPN. This study aims to detect and compare the hemodynamic characteristics of plantar blood vessels in patients with mild DPN and healthy participants to explore a simple and reliable new idea and a potential method for early assessment of DPN and to investigate the influence of gender and age on hemodynamic characteristics.

Research design and methods Sixty age-matched and gender-matched patients with mild DPN (30 men and 30 women) and 60 healthy participants were randomly recruited. Color Doppler ultrasound was used to measure and analyze the hemodynamic characteristics of plantarrelated vessels.

Results Ultrasonic measurements had good test-retest reliability. There may be no statistically significant differences in the blood flow velocity and blood flow in the plantar-related blood vessels of participants, irrespective of their gender and age. For patients with mild DPN, color Doppler ultrasound may indicate early hemodynamic abnormalities when there are no obvious abnormalities in the large arteries of the lower limbs, which are specifically manifested as increased blood flow velocity and blood flow in the distal small vessels.

Conclusions Our study provides in vivo data support for the dynamic characteristics of the plantar blood vessel biomechanical model and provides a new idea of in vivo and non-invasive early diagnosis of DPN.

\section{INTRODUCTION}

Diabetes is one of the common chronic endocrine diseases, which adversely affects the quality of life and even the patient's life span. In recent years, with the changes in an individual's lifestyle, environment, dietary structure, and global ageing, the incidence of diabetes has increased significantly, and a trend of younger age at diabetes onset has been observed. According to the latest report of the International Diabetes Federation in 2019, globally, approximately 463 million adults aged 20-79 years were diabetic (1 in 11 people were diabetic), which by 2030 , the

\section{Significance of this study}

What is already known about this subject?

- Some studies have shown that vascular changes and damage to the inner wall of blood vessels can damage the normal function and structure of peripheral nerves, thereby inducing diabetic peripheral neuropathy (DPN).

What are the new findings?

- For patients with DPN with mild neuropathy, if the color Doppler ultrasound examination of the lower extremity arteries does not reveal obvious morphological abnormalities, early hemodynamic abnormalities have appeared in small blood vessels.

- DPN with mild neuropathy has increased blood flow velocity and blood flow in the small blood vessels of the lower extremities.

- There may be no statistically significant difference in the blood flow velocity and blood flow of the plantarrelated blood vessels between gender and age.

How might these results change the focus of research or clinical practice?

- When the clinical symptoms of DPN are mild, the management of the blood vessels in the foot may be a promising management model for diabetic complications, which can be used to improve the early recognition of DPN or delay the disease progression.

- These findings support more foot blood flow tests in patients with DPN, including a larger patient population, while intervening in DPN and trying new research methods.

number is expected to reach 578.4 million, and by 2045, 700.2 million people might have diabetes. This increase will inevitably be accompanied by an increase in the prevalence of diabetic complications. ${ }^{1}$

Among the many complications, diabetic peripheral neuropathy (DPN) is more common. Its clinical manifestations are diverse, such as mild limb pain, numbness, paresthesia, and intermittent fatigue. Severe cases may suffer from ischemic gangrene of the lower limbs and may risk amputation. ${ }^{2}$ 
Patients with DPN are 10-20 times more likely to have an amputation than those without. Every $30 \mathrm{~s}$, a patient with DPN has their lower limbs or part of their lower limbs amputated. ${ }^{3}$ Therefore, early identification and treatment of DPN are crucial. Presently, detection methods and diagnostic standards of DPN have not been unified, which affects clinical diagnosis and treatment. ${ }^{4}$ Thus, it is necessary to find a simple, convenient, fast and reliable method for early diagnosis of DPN.

Presently, the mechanism of DPN is not very clear. The mechanism was once thought to be related to vascular dysfunction, metabolic disorders, neurotrophic factor deficiency, immune mechanism abnormalities, oxidative stress, etc. It is currently considered to be the result of the combined effects of various factors, among which vascular and metabolic factors are the most important. ${ }^{56}$ Some studies have reported that oxidative stress and metabolic disorders can cause vascular changes and damage to the inner wall of blood vessels, resulting in damage to the structure and function of peripheral nerves and inducing DPN. These studies provide ideas for early DPN screening. ${ }^{7-9}$ Some researchers have also confirmed that patients with DPN have impaired vasodilation and abnormal vascular morphology. ${ }^{10}{ }^{11}$ Abnormal structural morphology parameters will inevitably be accompanied by changes in vascular dynamic performance parameters (blood pressure, blood flow velocity, blood flow, etc). Nabuurs-Franssen $e t a l^{2}$ reported that the capillary blood flow in the dorsum of the feet of patients with DPN was lower than that of healthy volunteers. Pfützner $e t a l \mathrm{~s}^{13}$ findings confirmed the relationship between small nerve fiber injury and skin microvascular dysfunction. Park $e t$ $a l^{14}$ found that the positive peak perfusion and thermal hyperemia of the DPN group were significantly lower than that of the healthy control group and the diabetic control group without DPN. Archer et $a l^{15}$ found that the resting blood flow in the feet of patients with DPN was five times that in healthy participants' feet. These studies provide valuable information for a further study of vascular dynamic characteristics of DPN. However, there is no final conclusion about the changes and influencing factors of hemodynamic characteristics in patients with DPN.

Based on color Doppler ultrasound technology, the hemodynamic characteristics of plantar-related blood vessels in healthy individuals and patients with mild DPN were measured and compared, and the influence of age and gender on hemodynamic characteristics was investigated.

\section{METHOD}

\section{Participant selection}

This study randomly recruited 60 patients with type 2 diabetes and DPN in the Department of Neurology of the Second Hospital of Jilin University. An experienced neurologist confirmed the diagnosis of DPN with a 'diagnosed' clinical level. ${ }^{16}$ DPN was classified according to the Toronto Clinical Scoring System (TCSS) as 'probable neuropathy'. ${ }^{1617}$ TCSS score standard: $0-5$ points indicate no DPN; 6-8 points, mild DPN; 9-11 points, moderate DPN; and 12-19 points, severe DPN. In our study, the first step involved selecting patients with mild DPN with a TCSS score of 6-8. In the second step, patients with mild DPN underwent a vibration perception threshold (VPT) test, and patients with mild DPN with VPT results (range, 15-20) were selected and finally enrolled in the study. All tests were completed by experienced neurologists. ${ }^{18}$ The group without diabetes randomly recruited 60 volunteers corresponding to their age (volunteers meet the requirement of past fasting blood glucose level of $<7.0 \mathrm{mmol} / \mathrm{L}$, random blood glucose level of $<11.0 \mathrm{mmol} / \mathrm{L}$, glycohemoglobin (HbAlc) <6.5\%, no clear diagnosis or suspected diagnosis of diabetes). All participants in our study confirmed that they agreed to participate in the trial voluntarily.

Exclusion criteria involved patients with an anklebrachial index (ABI) of $<0.9$ at rest, a toe-brachial index of $<0.6$, or a decrease in the ABI by $20 \% 1 \mathrm{~min}$ after exercise, or with atherosclerotic plaque formation, arterial stenosis, or occlusion in the lower extremity confirmed using Doppler ultrasound. Additionally, patients with a history of amputation of both lower limbs, plantar fasciitis, foot infection, plantar warts, lumbar spinal stenosis, athlete's foot or a foot ulcer, recent vigorous physical activity, and a history of being overweight were also excluded.

Participants with mild DPN and those without diabetes were divided into the following three groups according to age: group I ( $40-49$ years old), group II ( $50-59$ years old), and group III ( $>60$ years old). There were 20 people in each group: 10 men and 10 women. The basic information of participants is presented in table 1 .

\section{Experimental equipment and protocol}

With reference to plantar vascular anatomy ${ }^{17}$ and hemodynamics, ${ }^{19}$ our study evaluated the six major arteries that supply blood to the foot sole, namely the first common plantar artery (FiCPDA), second common plantar artery (SCPDA), third common plantar artery (TCPDA), fourth common plantar artery (FoCPDA), posterior tibial artery (PTA), and fibular side of the first plantar toe proper artery (FPPDA). In the study, using an ultrasound imaging system (Aixplorer; SuperSonic Imagine, France) and a 2-10 MHz linear array broadband ultrasound probe, the hemodynamic parameters of the six arteries of each participant's foot were measured in the supine position (figure 1A). To keep participants steady, they were asked to lie down and rest for $10 \mathrm{~min}$ before the test and to keep their bodies relaxed throughout the test. At the same time, the temperature of the test room was maintained at $25^{\circ} \mathrm{C}$ to eliminate the influence of temperature on measurement results. The relative humidity was controlled at $20 \%-60 \%$, and all tests were completed within 30 days of the same season. 


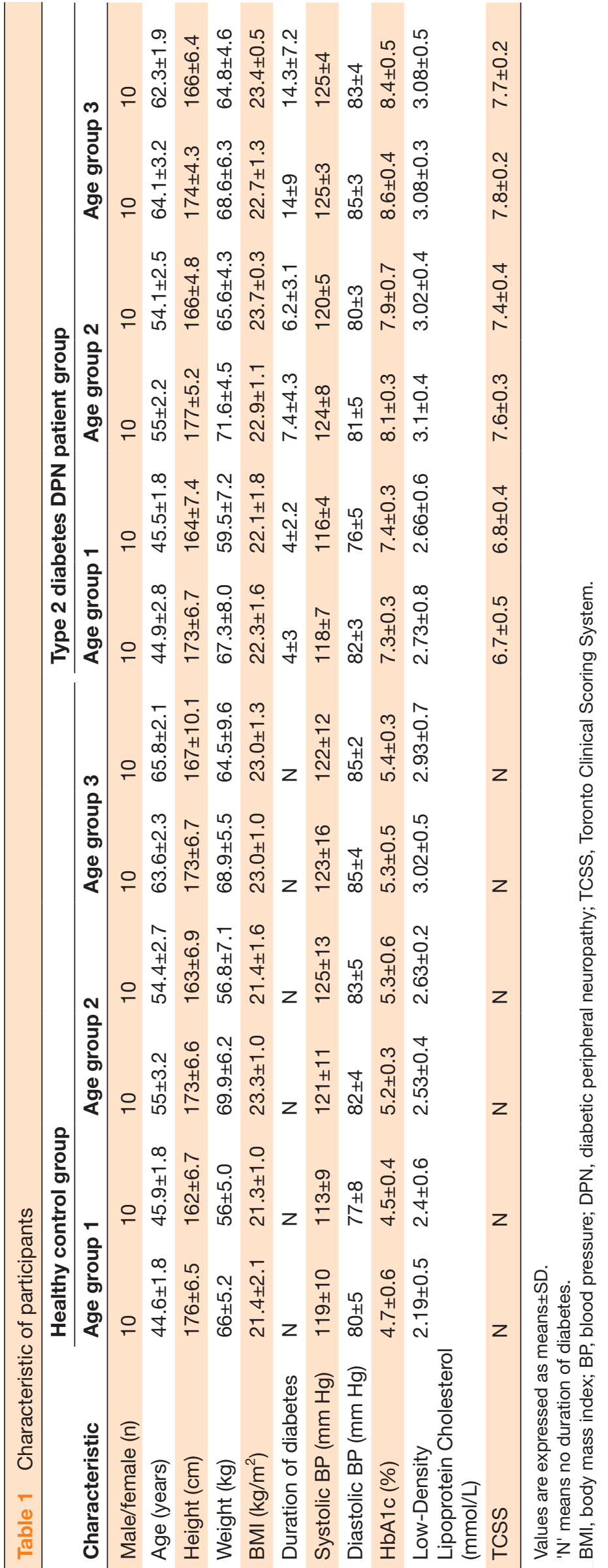




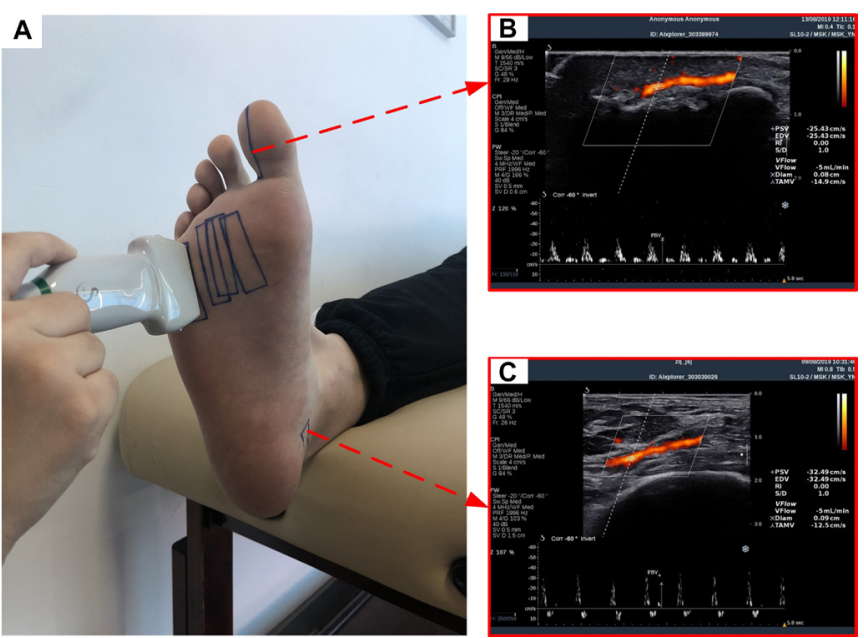

Figure 1 (A) Test position of the subject. (B) Typical color Doppler image of fibular proper plantar digital artery of the first toe. (C) Typical color Doppler image of posterior tibial artery.

With the help of the anatomical position, six arteries were located and measured to ensure consistency of the participant's detected positions. For instance, for evaluating the FPPDA, the ultrasound probe was located on the fibular side of the first toe, and the $\mathrm{S}$ end of the probe was facing the far end of the plantar. The probe angle should be adjusted, and the bony bulge of the distal phalanx of the first toe should be observed in the collected images. When the cortical bone was clearly displayed, the blood flow signal was collected on the left side of the cortical bone (figure 1B). The peak blood flow velocity and blood flow during systole were selected for analysis. ${ }^{20-22}$ The hemodynamic parameters of each blood vessel were measured three times by two doctors with $>3$ years of ultrasonic clinical testing experience.

\section{Data analyses}

The analyses of the measurement data of all hemodynamic parameters were performed using SPSS Statistics software V.21.0 (SPSS). As recommended by Hopkins, ${ }^{23}$ repeatability of the blood flow and its velocity in plantar vessels were assessed using intraclass correlation coefficient $(3,1)\left(\mathrm{ICC}_{3,1}\right)$, two-way random, absolute agreement, with $95 \%$ CI. It is generally believed that the repeatability of data measurement is poor when ICC ranges from 0 to 0.40 ; in the ranges of $0.41-0.60$ and $0.61-0.79$, the repeatability of data measurement was moderate and good. In the range of $0.8-1.0$, the repeatability of data measurement was excellent. ${ }^{23}$ The differences in hemodynamic parameters between the mild DPN group and the group without diabetes were analyzed with variance analysis of multiple comparisons using the general linear model method, and the effects of age and gender on hemodynamic characteristics were discussed. A $p$ value $<0.05$ was considered statistically significant. The measured values of hemodynamic parameters were expressed as mean \pm SD.
RESULTS

Repeatability analysis of measurement of hemodynamic parameters

The repeatability of vascular blood flow velocity and blood flow measurements is presented in online supplemental table S1. The results indicated that the $\mathrm{ICC}_{3,1}$ of blood flow velocity ranged from 0.964 to 0.991 (95\% CI 0.967 to 0.996$)$, and the $\mathrm{ICC}_{3,1}$ of blood flow ranged from 0.950 to 0.985 (95\% CI 0.953 to 0.991 ).

\section{Effects of gender and age on hemodynamic parameters}

The hemodynamic parameters based on gender are summarized in tables 2 and 3 . There were no statistically significant differences in the blood flow velocity and blood flow in plantar-related blood vessels among men and women, irrespective of whether participants had mild DPN or were healthy.

The hemodynamic parameters of three different age groups are presented in online supplemental tables S2 and S3. There were no statistically significant differences in the blood flow velocity and blood flow in the footrelated blood vessels among three different age groups, irrespective of whether participants had mild DPN or were healthy.

\section{Effect of DPN on hemodynamic parameters}

The hemodynamic parameters of the mild DPN group and the group without diabetes are presented in table 4 . Except for the PTA, the blood flow velocity and blood flow of other blood vessels were significantly different between the mild DPN group and the group without diabetes $(p<0.001)$, and the blood flow velocity and blood flow were greater in the mild DPN group than in the group without diabetes.

\section{DISCUSSION}

The onset of DPN is often occult, and the progress of the disease is slow. At the onset, patients often have no conscious symptoms. When the clinical symptoms manifest, the peripheral nerve has irreversible damage with corresponding pathological changes. Therefore, the early evaluation of DPN is crucial. If DPN is diagnosed early, active control of blood glucose level could be achieved, and necessary foot care could be administered in time; thus, foot ulcers, gangrene, amputation, and other serious consequences could be avoided..$^{24}$ DPN is a length-dependent neuropathy in which the damage of the epidermal axon of the distal lower extremity precedes the damage of the axon of the proximal lower extremity. Dyck $e t a l^{25}$ showed that the distribution of nerve damage in DPN is limb specific, and the lower limbs are affected earlier in the course of the disease. The neurophysiological evaluation of the distal branches of the sensory nerves of the lower extremities (such as the plantar nerves), in theory, may increase the sensitivity of nerve conduction study diagnosis, thereby allowing early DPN diagnosis. ${ }^{26}{ }^{27}$ Consistently, even in the early stages of the disease, neurophysiological examination of the distal 
Table 2 Blood flow velocity of blood vessels between different genders (unit: $\mathrm{cm} / \mathrm{s}$ )

\begin{tabular}{|c|c|c|c|c|c|c|c|}
\hline \multirow[b]{2}{*}{ Vessels } & \multirow[b]{2}{*}{ Gender } & \multicolumn{3}{|l|}{ Healthy } & \multicolumn{3}{|l|}{ DPN } \\
\hline & & Age group 1 & Age group 2 & Age group 3 & Age group 1 & Age group 2 & Age group 3 \\
\hline \multirow[t]{2}{*}{ FPPDA } & Male & $12.96 \pm 4.08$ & $15.81 \pm 4.94$ & $17.90 \pm 4.23$ & $25.22 \pm 3.70$ & $29.73 \pm 8.11$ & $27.64 \pm 8.27$ \\
\hline & Female & $15.48 \pm 1.67$ & $14.65 \pm 3.96$ & $18.14 \pm 4.69$ & $24.67 \pm 4.14$ & $24.58 \pm 3.71$ & $26.18 \pm 5.88$ \\
\hline \multirow[t]{2}{*}{ FiCPDA } & Male & $16.01 \pm 5.04$ & $18.01 \pm 3.00$ & $19.63 \pm 4.12$ & $29.21 \pm 6.84$ & $28.97 \pm 7.93$ & $25.43 \pm 6.28$ \\
\hline & Female & $17.01 \pm 3.06$ & $17.22 \pm 3.97$ & $19.63 \pm 3.35$ & $27.27 \pm 4.04$ & $27.38 \pm 5.99$ & $26.46 \pm 4.60$ \\
\hline \multirow[t]{2}{*}{ SCPDA } & Male & $17.81 \pm 2.36$ & $19.79 \pm 2.79$ & $17.49 \pm 2.87$ & $27.15 \pm 8.51$ & $26.28 \pm 4.42$ & $24.24 \pm 5.57$ \\
\hline & Female & $18.70 \pm 2.76$ & $19.48 \pm 3.84$ & $18.84 \pm 4.31$ & $24.12 \pm 1.56$ & $27.47 \pm 5.22$ & $24.28 \pm 5.22$ \\
\hline \multirow[t]{2}{*}{ TCPDA } & Male & $21.66 \pm 4.02$ & $21.54 \pm 3.01$ & $24.12 \pm 3.82$ & $33.13 \pm 8.16$ & $34.04 \pm 6.68$ & $30.77 \pm 7.70$ \\
\hline & Female & $21.38 \pm 4.33$ & $22.69 \pm 4.51$ & $25.07 \pm 3.54$ & $28.00 \pm 5.08$ & $29.75 \pm 3.73$ & $30.72 \pm 5.23$ \\
\hline \multirow[t]{2}{*}{ FoCPDA } & Male & $19.19 \pm 7.31$ & $20.25 \pm 3.99$ & $23.69 \pm 6.51$ & $30.45 \pm 4.69$ & $27.64 \pm 5.579$ & $31.52 \pm 8.38$ \\
\hline & Female & $20.42 \pm 4.62$ & $19.96 \pm 5.82$ & $19.63 \pm 4.28$ & $27.69 \pm 4.66$ & $25.83 \pm 3.18$ & $27.54 \pm 7.72$ \\
\hline \multirow[t]{2}{*}{ PTA } & Male & $30.86 \pm 6.61$ & $32.18 \pm 7.71$ & $35.69 \pm 9.76$ & $32.53 \pm 3.54$ & $37.09 \pm 10.18$ & $34.49 \pm 12.19$ \\
\hline & Female & $32.12 \pm 3.97$ & $29.89 \pm 3.98$ & $36.03 \pm 6.12$ & $33.27 \pm 8.30$ & $34.75 \pm 5.71$ & $41.57 \pm 15.39$ \\
\hline
\end{tabular}

Values are expressed as means \pm SD.

The multiple comparisons were carried out for the statistical analysis. One comparison was made between the genders ( $p=0.05$ ).

DPN, diabetic peripheral neuropathy; FiCPDA, first common plantar artery; FoCPDA, fourth common plantar artery; FPPDA, first plantar toe proper artery; PTA, posterior tibial artery; SCPDA, second common plantar artery; TCPDA, third common plantar artery.

nerves of the lower limbs (the dorsal sural nerve and medial and lateral plantar nerves) may help demonstrate distal axonal degeneration. ${ }^{28}$ Galiero $e t ~ a l^{29}$ confirmed the ability of whole plantar nerve (WPN) conduction in the early diagnosis of DPN. The study showed that the plantar nerve is affected earlier in the development of DPN than other peripheral nerves, resulting in the development of corresponding lesions. Therefore, according to the vascular anatomy and hemodynamics, our study evaluated six main arteries of the plantar and the hemodynamic characteristics under the condition of whole-body relaxation of the participants and explored the changes in plantar-related hemodynamic parameters in patients with mild DPN and healthy controls. Our study provides a richer theoretical basis for the pathogenesis of DPN and the study of foot hemodynamics and provides new ideas and data support for the early evaluation and diagnosis of clinical DPN.

Our study evaluated the accuracy of color Doppler ultrasound measurement of the hemodynamic parameters of plantar-related blood vessels using remeasurement reliability. For all blood flow and blood flow velocity

Table 3 Blood flow of blood vessels between different genders (unit: $\mathrm{mL} / \mathrm{min}$ )

\begin{tabular}{|c|c|c|c|c|c|c|c|}
\hline \multirow[b]{2}{*}{ Vessels } & \multirow[b]{2}{*}{ Gender } & \multicolumn{3}{|l|}{ Healthy } & \multicolumn{3}{|l|}{ DPN } \\
\hline & & Age group 1 & Age group 2 & Age group 3 & Age group 1 & Age group 2 & Age group 3 \\
\hline \multirow[t]{2}{*}{ FPPDA } & Male & $1.20 \pm 0.42$ & $1.20 \pm 0.63$ & $1.40 \pm 0.70$ & $2.37 \pm 0.67$ & $2.77 \pm 0.82$ & $2.60 \pm 1.39$ \\
\hline & Female & $1.30 \pm 0.48$ & $1.30 \pm 0.48$ & $1.60 \pm 0.70$ & $2.13 \pm 1.07$ & $2.53 \pm 1.06$ & $3.17 \pm 0.82$ \\
\hline \multirow[t]{2}{*}{ FiCPDA } & Male & $1.50 \pm 0.53$ & $1.30 \pm 0.48$ & $1.50 \pm 0.71$ & $2.63 \pm 0.76$ & $2.64 \pm 1.11$ & $2.23 \pm 0.82$ \\
\hline & Female & $1.50 \pm 0.71$ & $1.30 \pm 0.48$ & $1.80 \pm 0.92$ & $2.60 \pm 1.24$ & $3.03 \pm 0.71$ & $2.70 \pm 0.73$ \\
\hline \multirow[t]{2}{*}{ SCPDA } & Male & $1.40 \pm 0.70$ & $1.30 \pm 0.48$ & $1.40 \pm 0.52$ & $2.30 \pm 0.74$ & $2.43 \pm 1.25$ & $2.67 \pm 1.49$ \\
\hline & Female & $1.30 \pm 0.48$ & $1.30 \pm 0.48$ & $1.70 \pm 0.67$ & $2.13 \pm 1.07$ & $2.77 \pm 0.63$ & $2.90 \pm 1.21$ \\
\hline \multirow[t]{2}{*}{ TCPDA } & Male & $1.50 \pm 0.52$ & $1.20 \pm 0.42$ & $1.80 \pm 0.79$ & $2.53 \pm 0.36$ & $2.63 \pm 1.29$ & $2.73 \pm 0.89$ \\
\hline & Female & $1.30 \pm 0.67$ & $1.40 \pm 0.70$ & $1.40 \pm 0.52$ & $2.17 \pm 0.53$ & $2.40 \pm 0.62$ & $2.17 \pm 0.98$ \\
\hline \multirow[t]{2}{*}{ FoCPDA } & Male & $1.30 \pm 0.48$ & $1.30 \pm 0.67$ & $1.50 \pm 0.71$ & $3.23 \pm 1.34$ & $2.60 \pm 1.36$ & $2.93 \pm 1.77$ \\
\hline & Female & $1.60 \pm 0.70$ & $1.10 \pm 0.32$ & $1.70 \pm 0.82$ & $3.47 \pm 1.90$ & $2.94 \pm 1.89$ & $2.967 \pm 1.54$ \\
\hline \multirow[t]{2}{*}{ PTA } & Male & $3.80 \pm 2.20$ & $2.80 \pm 1.69$ & $4.30 \pm 2.06$ & $3.40 \pm 0.98$ & $4.17 \pm 1.94$ & $4.07 \pm 1.61$ \\
\hline & Female & $4.00 \pm 2.45$ & $2.50 \pm 1.08$ & $3.40 \pm 1.07$ & $3.10 \pm 1.64$ & $3.40 \pm 1.58$ & $2.67 \pm 3.37$ \\
\hline
\end{tabular}

Values are expressed as means \pm SD.

The multiple comparisons were carried out for the statistical analysis. One comparison was made between the genders ( $p=0.05$ ).

DPN, diabetic peripheral neuropathy; FiCPDA, first common plantar artery; FoCPDA, fourth common plantar artery; FPPDA, first plantar toe proper artery; PTA, posterior tibial artery; SCPDA, second common plantar artery; TCPDA, third common plantar artery. 
Table 4 Blood flow velocity (unit: $\mathrm{cm} / \mathrm{s}$ ) and volume (unit: $\mathrm{mL} / \mathrm{min}$ ) of blood vessels between DPN and healthy control group

\begin{tabular}{llll}
\hline Vessels & Gender & $\begin{array}{l}\text { Blood flow } \\
\text { velocity }\end{array}$ & $\begin{array}{l}\text { Blood flow } \\
\text { volume }\end{array}$ \\
\hline FPPDA & Healthy & $15.83 \pm 4.45$ & $1.33 \pm 0.60$ \\
& DPN & $26.34 \pm 6.24^{*}$ & $2.60 \pm 1.05^{\star}$ \\
FiCPDA & Healthy & $17.92 \pm 4.05$ & $1.48 \pm 0.68$ \\
& DPN & $27.45 \pm 6.23^{\star}$ & $2.64 \pm 0.95^{\star}$ \\
SCPDA & Healthy & $18.69 \pm 3.33$ & $1.4 \pm 0.58$ \\
& DPN & $25.59 \pm 5.66^{*}$ & $2.53 \pm 1.14^{*}$ \\
\hline TCPDA & Healthy & $22.76 \pm 4.15$ & $1.43 \pm 0.65$ \\
& DPN & $31.07 \pm 6.61^{*}$ & $2.44 \pm 0.86^{\star}$ \\
FoCPDA & Healthy & $20.52 \pm 5.75$ & $1.42 \pm 0.67$ \\
& DPN & $28.45 \pm 6.29^{*}$ & $3.02 \pm 1.67^{*}$ \\
\hline PTA & Healthy & $32.63 \pm 6.99$ & $3.47 \pm 1.95$ \\
& DPN & $35.62 \pm 10.47$ & $3.8 \pm 2.07$ \\
\hline
\end{tabular}

Values are expressed as means \pm SD.

The multiple comparisons were carried out for the statistical analysis. DPN and the group without diabetes were compared once.

${ }^{*}$ There is significant difference between DPN and the group without diabetes $(p<0.05)$.

DPN, diabetic peripheral neuropathy; FiCPDA, first common plantar artery; FoCPDA, fourth common plantar artery; FPPDA, first plantar toe proper artery; PTA, posterior tibial artery; SCPDA, second common plantar artery; TCPDA, third common plantar artery.

measurements, the ICC and $95 \%$ CI ranged from 0.950 to 0.991 and 0.953 to 0.996 , respectively. Our study shows excellent test-retest reliability in the ultrasonic detection results of systemic vascular blood flow and blood flow velocity, which also shows that color Doppler ultrasound is a reliable and repeatable technology. In measuring the hemodynamic parameters of plantar vessels, it may become an important tool for studying the pathogenesis and early diagnosis of DPN.

Our patients with type 2 diabetes with mild DPN and the healthy participants had normal body mass index (BMI). The BMI of all participants was between 18.5 and $24.9 \mathrm{~kg} / \mathrm{m}^{2}$; however, there was no significant difference. The difference in test results caused by obesity was excluded. Previous studies on the lower extremity arteries of participants focused mainly on main blood vessels of the lower extremities, such as the PTA, femoral artery, dorsal foot artery, etc. There are few reports on the blood supply arteries of the front plantar region. Guirro $e t a t^{20}$ studied the blood flow velocity of the lower extremity arteries in diabetic women. The study found that the peak blood flow velocity in the PTA of healthy individuals was $55.47 \pm 13.06 \mathrm{~cm} / \mathrm{s}$. Fronek $e t a l^{21}$ also reported that the peak blood flow velocity in the PTA of healthy individuals was $16.0 \pm 10.0 \mathrm{~cm} / \mathrm{s}$. In our study, the peak blood flow velocity in the PTA lies between the values reported in the previous two studies. The reason for this difference may be related to the different measurement positions of the tested blood vessels. Moreover, it is well known that even for the same blood vessel, participants' different exercise states, different body positions, and other factors will result in differences in vascular hemodynamic parameters. Therefore, considering the differences in the distribution characteristics of vascular hemodynamic parameters, ${ }^{30-32}$ the probe and measurement position were strictly defined in our study. In previous studies, it was reported that the degree of stenosis of blood vessels directly affects the blood flow velocity. ${ }^{24}{ }^{33}$ Bonesi et $a \hat{l}^{34}$ used Doppler optical coherence tomography noninvasively to monitor the blood flow velocity distribution in blood vessels with complex geometric shapes in vivo. The study found that the blood flow velocity distribution was non-uniform and closely related to the geometry of the blood vessel. Therefore, to avoid measurement errors caused by differences in the shape and direction of the plantar blood vessels, consistency between measurement positions for different participants was guaranteed. Based on the vascular anatomy atlas, the cortical bone structure of calcaneus, phalanx, and metatarsal bones was selected to locate and measure six arterial blood vessels. With the PTA as an example, the probe was placed $2.5 \mathrm{~cm}$ inferior to the medial malleolus protrusion. With the B-mode ultrasound, the PTA was visible, and the cortical structure of the calcaneus was placed at the lower right boundary of the imaging area. By fine tuning the direction of the ultrasonic probe, data collection of blood flow and blood flow velocity was performed when the PTA was clearly visible with a stable blood flow signal (figure 1C). Thus, our study guaranteed the consistency of measurement positions between different participants and operators.

Regarding the influence of gender on the hemodynamic characteristics of plantar-related blood vessels, there was no significant statistical difference in the blood flow velocity and blood flow of plantar-related blood vessels between men and women, irrespective of whether participants had mild DPN or were healthy. The $p$ values of the statistical test of the dynamic parameters of blood flow among men and women are presented in online supplemental tables S4 and S5. Regarding the influence of age on the hemodynamic characteristics of plantarrelated blood vessels, there was no statistically significant difference in the blood flow velocity and blood flow of plantar-related blood vessels in different genders, irrespective of whether participants had mild DPN or were healthy. The $p$ values of the statistical test of blood flow dynamic parameters among three different age groups are presented in online supplemental tables S6 and S7. Although our results indicated that gender and age have no effect on the hemodynamic characteristics of plantarrelated blood vessels, it does not indicate that the hemodynamic characteristics of blood vessels were not changed. The reason for the analysis may be related to selection and number of participants analyzed here. For participants, arteriosclerosis caused by age and gender ${ }^{35} 36$ has not reached the level of causing significant changes in 
the hemodynamic characteristics of blood vessels; it may also be related to the small sample size, measurement location, and other factors. Therefore, the influence of gender and age on the hemodynamic characteristics of plantar-related blood vessels remains to be further studied. To study the influence of age on the hemodynamic characteristics of foot-related vessels, the selection of age group was considered in our study. In a previous study, Jan $e t a l^{37}$ reported that for healthy volunteers, the difference in age may not cause a significant effect of ageing on microvascular dysfunction. ${ }^{37} \mathrm{~A}$ previous study also demonstrated that significant changes related to ageing were observed for patients over 65 years old. ${ }^{38}$ Sun $e t a l^{9}$ explored the correlation between the microvascular microcirculation and DPN severity in patients aged 50-60 years. Viswanathan $e t a t^{40}$ evaluated the blood supply of lower limbs in patients with DPN aged 60-76 based on transcutaneous oxygen tension. In addition to the studies of single-age group, some age group divisions covered the typical and important stages of human life, which is of great significance to the study of the whole process of human life; however, these age divisions have a large span for each age group and lack some middleaged data. Therefore, based on previous studies, our study divides all participants into groups by age at 10 -year intervals, filling the gaps in previous studies that lack part of the middle-aged plantar-related vascular dynamic parameters.

Additionally, our study examined and compared the plantar-related vascular dynamic characteristics of patients with mild DPN and healthy controls and explored the effect of DPN on hemodynamic parameters. Our results indicate that PTA is a major blood vessel that supplies the sole of the plantar, and FiCPDA, SCPDA, TCPDA, FoCPDA, and FPPDA belong to the small vessels of the plantar artery branch. In the early period of DPN, when the hemodynamic parameters of the large vessel did not change significantly, the blood vessel velocity and blood flow in small vessels of the plantar artery branch changed, indicating that the changes in the local small blood vessel developed before the lesions of the plantar large blood vessels. This may be owing to a compensatory physiological response because the heat dissipation process, owing to increased metabolism, is achieved by accelerating the blood flow. ${ }^{41}$ It is also possible that owing to the hypercoagulable state of blood in patients with diabetes, the deposition of glycosylation end products leads to stenosis of the vascular lumen, and the early-stage DPN mainly involves the small vessels. When blood flows through relatively narrow small blood vessels, the blood flow signal becomes weaker, and the flow rate becomes constant, resulting in faster blood flow. With the aggravation of the disease, the body's hyperglycemia state accelerates the process of forming end-glycosylation products. The increased number of end-glycosylation products leads to accumulation, which in turn induces various pathological changes, thickens the blood vessel wall, and reduces blood vessel elasticity after the proliferation of smooth muscle cells. Mononuclear macrophages and smooth muscle cells induce a lipid reaction that leads to plaque formation, further narrowing or even occluding the blood vessel lumen, and reduced blood flow, which ultimately leads to a series of clinical manifestations of ischemia. ${ }^{42}$ Although the results of our study show that in patients with mild DPN, the color Doppler ultrasound examination of the lower extremities does not indicate obvious abnormalities, the small blood vessels already appear to have early hemodynamic abnormalities, including the increase in blood flow velocity and blood flow. However, this study only conducted a crosssectional study on patients and could not absolutely infer the causal relationship between the results and mild DPN. At the same time, with known correlations between different complications of diabetes, especially in the early stage, ${ }^{43}$ for example, using color Doppler ultrasound technology, fundus examination, assessment of kidney function in terms of estimated glomerular filtration rate, and estimation of kidney damage in terms of albuminuria, ${ }^{44} 45$ it may be possible to evaluate the relationship between early DPN and complications such as diabetic retinopathy and diabetic nephropathy. Therefore, our findings can help identify early signs of neuropathy and may identify or exclude other complications of diabetes, such as diabetic retinopathy, diabetic nephropathy, etc. ${ }^{43}$

The color Doppler ultrasound technology was used in our study to detect and compare the hemodynamic characteristics of foot-related vessels in the mild DPN group and the group without diabetes and to explore the influence of gender and age on hemodynamic characteristics, which is of great significance. Our study provides the in vivo data support for the dynamic characteristics of foot hemodynamic modeling and provides more information for early clinical evaluation and diagnosis.

However, our study has certain limitations. First, this was a cross-sectional study. Presently, it is not possible to infer the causal relationship between our findings and mild DPN absolutely. In follow-up research, with more participants, the hemodynamic characteristics of the plantar blood vessels can be tracked and monitored longitudinally to identify the trends and factors of hemodynamic characteristics during the occurrence and development of DPN, which may be helpful towards the prevention and treatment of DPN and serious foot complications. Second, the sample size of our study was limited to 120 cases (60 patients and 60 healthy controls). Thus, a larger population is needed to determine whether there are differences between different age groups and gender groups. Third, except for the PTA, the major artery supplying blood to the plantar, the other five arteries evaluated in the study were all distributed in the sole of the plantar. Thus, it is essential to compare the hemodynamic characteristics of the dorsal foot-related vessels between the mild DPN group and the group without diabetes in the future. Fourth, recent studies have shown that diabetic plantar neuropathy can be diagnosed with WPN conduction. WPN testing needs to be included in 
follow-up studies to facilitate further research and evaluation of the correlation between WPN and early recognition of plantar blood vessels in patients with diabetes. Last, only patients with mild DPN with a TCSS score of 6-8 were selected in our study because some studies have confirmed that the severity of DPN will affect the vascular microcirculation. ${ }^{39}$ Hence, future studies should clarify the impact of DPN severity on hemodynamic characteristics.

\section{CONCLUSIONS}

In summary, our study provides data support for the dynamic characteristics of blood vessel biomechanics modeling in the foot and provides a new idea of noninvasive, simple, convenient, and rapid early assessment of DPN for clinical use. The color Doppler ultrasound technology is a reliable and repeatable technique for measuring the hemodynamic parameters of plantar vessels. Regardless of gender and age, there may be no statistically significant differences in the blood flow velocity and blood flow of the blood vessels associated with the plantar of the participants. For patients with mild DPN, the early hemodynamic abnormalities, that is, the increase in blood flow velocity and blood flow, appeared in small vessels without obvious abnormalities in color Doppler ultrasound imaging of the lower extremity artery.

Contributors QZ and MY collected the data. JL and JW analyzed the data, all under the supervision of LeR (principal investigator) and LuR. JL drafted the manuscript. QZ and ZQ reviewed and edited the draft. LeR is the guarantor of this work and, as such, had full access to all the data in the study and takes responsibility for the integrity of the data and the accuracy of the data analysis.

Funding This work was supported by the National Key Research \& Development Program of China (grant numbers SQ2020YFB170274, 2018YFC2001300) and the National Natural Science Foundation of China (grant numbers 51905207, 52175270, 91948302, 91848204, 52021003, 52005209).

\section{Competing interests None declared.}

\section{Patient consent for publication Not required.}

Ethics approval This study involves human participants. All the testing procedures and methods were approved by the Ethics Committee of the Second Hospital of Jilin University (No 2021-187). Participants gave informed consent to participate in the study before taking part.

\section{Provenance and peer review Not commissioned; externally peer reviewed.}

Data availability statement All data relevant to the study are included in the article or uploaded as supplementary information.

Supplemental material This content has been supplied by the author(s). It has not been vetted by BMJ Publishing Group Limited (BMJ) and may not have been peer-reviewed. Any opinions or recommendations discussed are solely those of the author(s) and are not endorsed by BMJ. BMJ disclaims all liability and responsibility arising from any reliance placed on the content. Where the content includes any translated material, BMJ does not warrant the accuracy and reliability of the translations (including but not limited to local regulations, clinical guidelines, terminology, drug names and drug dosages), and is not responsible for any error and/or omissions arising from translation and adaptation or otherwise.

Open access This is an open access article distributed in accordance with the Creative Commons Attribution Non Commercial (CC BY-NC 4.0) license, which permits others to distribute, remix, adapt, build upon this work non-commercially, and license their derivative works on different terms, provided the original work is properly cited, appropriate credit is given, any changes made indicated, and the use is non-commercial. See: http://creativecommons.org/licenses/by-nc/4.0/.
ORCID iD

Lei Ren http://orcid.org/0000-0003-3222-2102

\section{REFERENCES}

1 Saeedi P, Petersohn I, Salpea P, et al. Global and regional diabetes prevalence estimates for 2019 and projections for 2030 and 2045: Results from the International Diabetes Federation Diabetes Atlas, $9^{\text {th }}$ edition. Diabetes Res Clin Pract 2019;157:107843.

2 Kerr M, Rayman G, Jeffcoate WJ. Cost of diabetic foot disease to the National health service in England. Diabet Med 2014;31:1498-504.

3 Faruque LI, Wiebe N, Ehteshami-Afshar A, et al. Effect of telemedicine on glycated hemoglobin in diabetes: a systematic review and meta-analysis of randomized trials. CMAJ 2017;189:E341-64.

4 Sanz-Corbalán I, Lázaro-Martínez JL, García-Morales E, et al. Advantages of early diagnosis of diabetic neuropathy in the prevention of diabetic foot ulcers. Diabetes Res Clin Pract 2018;146:148-54.

5 Ejaz S, Chekarova I, Ejaz A, et al. Importance of pericytes and mechanisms of pericyte loss during diabetes retinopathy. Diabetes Obes Metab 2008;10:53-63.

6 Cameron NE, Eaton SE, Cotter MA, et al. Vascular factors and metabolic interactions in the pathogenesis of diabetic neuropathy. Diabetologia 2001;44:1973-88.

7 Callaghan BC, Cheng HT, Stables CL, et al. Diabetic neuropathy: clinical manifestations and current treatments. Lancet Neurol 2012;11:521-34.

8 Menegazzo L, Albiero M, Avogaro A, et al. Endothelial progenitor cells in diabetes mellitus. Biofactors 2012;38:194-202.

9 Tentolouris A, Eleftheriadou I, Tzeravini E, et al. Endothelium as a therapeutic target in diabetes mellitus: from basic mechanisms to clinical practice. Curr Med Chem 2020;27:1089-131.

10 Malik RA, Tesfaye S, Newrick PG, et al. Sural nerve pathology in diabetic patients with minimal but progressive neuropathy. Diabetologia 2005;48:578-85.

11 Bönhof GJ, Herder C, Strom A, et al. Emerging biomarkers, tools, and treatments for diabetic polyneuropathy. Endocr Rev 2019;40:153-92.

12 Nabuurs-Franssen MH, Houben AJHM, Tooke JE, et al. The effect of polyneuropathy on foot microcirculation in type II diabetes. Diabetologia 2002;45:1164-71.

13 Pfützner A, Forst T, Engelbach $\mathrm{M}$, et al. The influence of isolated small nerve fibre dysfunction on microvascular control in patients with diabetes mellitus. Diabet Med 2001;18:489-94.

14 Park HS, Yun HM, Jung IM, et al. Role of laser Doppler for the evaluation of pedal microcirculatory function in diabetic neuropathy patients. Microcirculation 2016;23:44-52.

15 Archer AG, Roberts VC, Watkins PJ. Blood flow patterns in painful diabetic neuropathy. Diabetologia 1984;27:563-7.

16 Boulton AJM, Malik RA, Arezzo JC, et al. Diabetic somatic neuropathies. Diabetes Care 2004;27:1458-86.

17 Ramsey EM, Anatomy V. Biology of the uterus. US: Springer, 1977: 59-76.

18 Medakkel AA, Sheela P. Vibration perception threshold values and clinical symptoms of diabetic peripheral neuropathy. Jou Clin Dia Res 2018;12:LC20-3 https://www.jcdr.net/articles/PDF/11549/ 32825_CE[Ra1]_F(SHU)_PF1(AJ_SL)_PFA(AJ_SL)_PB(AJ_SL)_PN( SL).pdf

19 Westerhof N, Stergiopulos N, Noble MIM. Snapshots of hemodynamics. US: Springer, 2005: 18. 12-15.

20 Guirro ECdeO, Guirro RRdeJ, Dibai-Filho AV, et al. Decrease in Talocrural joint mobility is related to alteration of the arterial blood flow velocity in the lower limb in diabetic women. J Phys Ther Sci 2014;26:553-6.

21 Fronek A, Coel M, Berstein EF. Quantitative ultrasonographic studies of lower extremity flow velocities in health and disease. Circulation 1976;53:957-60

22 Zou C, Jiao Y, Li X, et al. Differences between healthy adults and patients with type 2 diabetes mellitus in reactivity of toe microcirculation by ultrasound combined with a warm Bath test. Medicine 2017;96:22.

23 Hopkins WG. Measures of reliability in sports medicine and science. Sports Med 2000;30:1-15.

24 Selvarajah D, Kar D, Khunti K, et al. Diabetic peripheral neuropathy: advances in diagnosis and strategies for screening and early intervention. Lancet Diabetes Endocrinol 2019;7:938-48.

25 Dyck PJ, Albers JW, Andersen H, et al. Diabetic polyneuropathies: update on research definition, diagnostic criteria and estimation of severity. Diabetes Metab Res Rev 2011;27:620-8. 
26 Kural MA, Karlsson P, Pugdahl K, et al. Diagnostic utility of distal nerve conduction studies and sural near-nerve needle recording in polyneuropathy. Clin Neurophysiol 2017;128:1590-5.

27 Sylantiev C, Schwartz R, Chapman J, et al. Medial plantar nerve testing facilitates identification of polyneuropathy. Muscle Nerve 2008;38:1595-8.

28 Frigeni B, Cacciavillani M, Ermani M, et al. Neurophysiological examination of dorsal sural nerve. Muscle Nerve 2012;46:891-4.

29 Galiero R, Ricciardi D, Pafundi PC. Whole plantar nerve conduction study: a new tool for early diagnosis of peripheral diabetic neuropathy. Diabetes Research and Clinical Practice 2008;38:1595-8.

30 Hollnagel DI, Summers PE, Kollias SS, et al. Laser Doppler velocimetry (LDV) and 3D phase-contrast magnetic resonance angiography (PC-MRA) velocity measurements: validation in an anatomically accurate cerebral artery aneurysm model with steady flow. J Magn Reson Imaging 2007;26:1493-505.

31 Sadeghi MR, Shirani E, Tafazzoli-Shadpour M, et al. The effects of stenosis severity on the hemodynamic parameters-assessment of the correlation between stress phase angle and wall shear stress. $J$ Biomech 2011;44:2614-26.

32 Sui B, Gao P, Lin Y, et al. Hemodynamic parameters distribution of upstream, stenosis center, and downstream sides of plaques in carotid artery with different stenosis: a MRI and CFD study. Acta Radiol 2015;56:347-54.

33 Report of a who consultation, 2000: obesity: preventing and managing the global epidemic. Report of a who consultation. World Health Organ Tech Rep Ser 2000;894:1-253.

34 Bonesi M, Churmakovi D, Meglinski I. Imaging of flow velocity profiles within the complex geometry vessels by Doppler Optical Coherence Tomography - art. no. 68550B. Conference on Complex Dynamics and Fluctuations in Biomedical Photonics V: 2008; 19-21 Jan 2008, San Jose, CA, 2008:1202-13.
35 Steppan J, Barodka V, Berkowitz DE, et al. Vascular stiffness and increased pulse pressure in the aging cardiovascular system. Cardio Res Pract 2011;2011:1-8.

36 Wen W, Peng B, Tang X, et al. Prevalence of high arterial stiffness and gender-specific differences in the relationships with classical cardiovascular risk factors. J Atheroscler Thromb 2015;22:706-17.

37 Jan Y-K, Liao F, Cheing GLY, et al. Differences in skin blood flow oscillations between the plantar and dorsal foot in people with diabetes mellitus and peripheral neuropathy. Microvasc Res 2019;122:45-51.

38 Shiogai Y, Stefanovska A, McClintock PVE. Nonlinear dynamics of cardiovascular ageing. Phys Rep 2010;488:51-110.

39 Sun P-C, Kuo C-D, Chi L-Y, et al. Microcirculatory vasomotor changes are associated with severity of peripheral neuropathy in patients with type 2 diabetes. Diab Vasc Dis Res 2013;10:270-6.

40 Viswanathan V, Kadiri M, Medimpudi S, et al. Association of non-alcoholic fatty liver disease with diabetic microvascular and macrovascular complications in South Indian diabetic subjects. Int $J$ Diabetes Dev Ctries 2010;30:208-12.

41 Tesfaye S, Malik R, Ward JD. Vascular factors in diabetic neuropathy. Diabetologia 1994;37:847-54.

42 El Boghdady NA, Badr GA. Evaluation of oxidative stress markers and vascular risk factors in patients with diabetic peripheral neuropathy. Cell Biochem Funct 2012;30:328-34.

43 pp Sasso FC, Salvatore T, Tranchino G, et al. Cochlear dysfunction in type 2 diabetes: a complication independent of neuropathy and acute hyperglycemia. Metabolism 1999;48:1346-50.

44 Tuttle KR, Bakris GL, Bilous RW, et al. Diabetic kidney disease: a report from an ADA consensus conference. Diabetes Care 2014;37:2864-83.

45 Lecleire-Collet A, Erginay A, Angioi-Duprez K, et al. [A new grading system from color fundus photographs for screening for diabetic retinopathy]. J Fr Ophtalmol 2007;30:674-87. 